\title{
Comparative analysis and implementation of structured edge active contour
}

\author{
Brijesh N. Shah ${ }^{1}$, Jaymin K. Bhalani ${ }^{2}$ \\ ${ }^{1}$ Department of Electronics and Communication Engineering, Charusat University, India \\ ${ }^{2}$ Department of Electronics and Communication Engineering, BIT Campus, India
}

\begin{tabular}{l} 
Article Info \\
\hline Article history: \\
Received May 9, 2019 \\
Revised Oct 19, 2019 \\
Accepted Oct 31, 2019 \\
\hline
\end{tabular}

Keywords:

Active Contour

Difference of Gaussian (DOG)

Image segmentation

Linear Structure Tensor

\begin{abstract}
This paper proposes modified chanvese model which can be implemented on image for segmentation. The structure of paper is based on Linear structure tensor (LST) as input to the variant model. Structure tensor is a matrix illustration of partial derivative information. In the proposed model, the original image is considered as information channel for computing structure tensor. Difference of Gaussian (DOG) is featuring improvement in which we can get less blurred image than original image. In this paper LST is modified by adding intensity information to enhance orientation information. Finally Active Contour Model (ACM) is used to segment the images. The proposed algorithm is tested on various images and also on some images which have intensity inhomogeneity and results are shown. Also, the results with other algorithms like chanvese, Bhattacharya, Gabor based chanvese and Novel structure tensor based model are compared. It is verified that accuracy of proposed model is the best. The biggest advantage of proposed model is clear edge enhancement.
\end{abstract}

Copyright $(0) 2020$ Institute of Advanced Engineering and Science. All rights reserved.

\section{Corresponding Author:}

Brijesh N Shah,

Department of Electronics and Communication Engineering,

Charusat University,

Changa, Gujrat, India.

Email: brijeshshah.ec@charusat.ac.in

\section{INTRODUCTION}

Image segmentation is very essential part of image processing. Basically in segmentation, we partition original image into several regions, which are useful from our application point of view. Since many decades several techniques of segmentation were projected. The Active contour Model (ACM) $[1,2]$ which was shown by Kass [3], has been demonstrated as good method for image segmentation. The general method for ACM is to start with initial contour then we can make some rule to find steady state condition. it means we can reach correct object boundary. In various publish papers ACM differ in how to select initial contour, various evolution mechanisms and at which time contour should be stopped from other evolution. The existing ACM $[4,5]$ can be divided into two main categories i.e. region based and edge based. Edge based methods used to capture object boundaries in curve evolution. The main problem with these methods is they work poorly in presence of noise. They cannot segment object when there is hazy boundary.

Another problem with these type of method is that in the beginning the contour must be fixed nearby the object, else it is very problematic to get proper segmentation result. Region based model mark complete usage of image geometric analysis as compared to edge based model. There are many benefits of region based segmentation over edge based segmentation like they are less responsive to noise. Also, they are not more profound to contour initialization process. In addition to these, they can easily segment images with weak boundaries. The famous region based method was proposed by Chan-Vese [6] which is based on Mumford shah function. The main assumption in $\mathrm{C}-\mathrm{V}$ model $[7,8]$ is that image intensities are homogeneous 
in each region. So, it would not be useful on images which have intensity inhomogeneity [9, 10]. For dense demonstration of texture Linear Structure Tensor can be used. Structure tensor can be calculated from spatial derivatives of the image.

LST Structure tensor [11] are matrix representation of partial derivative information It has more powerful descriptions of local patterns as opposed to the directional derivative through its coherence measure.it is useful in many applications like corner detection. For a gray scale image the matrix field of structure tensor is given by

$$
\begin{gathered}
J_{0}=\nabla h \nabla h^{T}=\left[\begin{array}{cc}
h_{x}^{2} & h_{x} h_{y} \\
h_{x} h_{y} & h_{y}^{2}
\end{array}\right] \\
\text { where } \nabla h=\left[\begin{array}{cc}
\frac{\partial h}{\partial x} & \frac{\partial h}{\partial y}
\end{array}\right]^{T}, h_{x}=\frac{\partial h}{\partial x}, h_{y}=\frac{\partial h}{\partial y} \\
\text { and } T=\text { Matrix transpose }
\end{gathered}
$$

For avoiding cancellation of opposite signed gradient, when direct integration is performed, gradient is considered as form of its outer product. In order to make matrix field more immune to noise, smoothing operation is performed by convolving matrix component with a Gaussian kernel $\mathrm{K} \sigma$ with standard deviation $\sigma$.

$$
J_{\sigma}=K_{\sigma} *\left(\nabla h \nabla h^{T}\right)
$$

Where, * indicate convolution operator. An LST model can be used for segmentation of texture images. The texture $[12,13]$ type of images which have intensity inhomogeneity cannot be segmented by this model. Therefore this paper suggest the solution of this problem with a combination of filter based tensor values to LST.

The chan-vese model

The Chan-Vese model for active contour is a method through which we are able to segment different types of images. The images which are not segmented by thresholding can also be segmented by this model. This model is based on Mumford Shah functional for segmentation which is widely used in image segmentation. This algorithm is also useful to segment the object which does not have clearly defined boundaries. This algorithm is based on level sets [14-16].

The chan-vese model can give solution by minimizing following energy function

$$
\begin{aligned}
E^{C V}\left(c_{1}, c_{2}, C\right) & =\mu . \text { Length }(C)+\lambda_{1} \cdot \int_{\text {inside }(C)}\left|u_{0}(x, y)-c_{1}\right|^{2} \\
& +\lambda_{2} \cdot \int_{\text {outside }(C)}\left|u_{0}(x, y)-c_{2}\right|^{2} d x d y
\end{aligned}
$$

Here, $\mu, \lambda_{1}$ and $\lambda_{2}$ are constant, generally $\lambda_{1}=\lambda_{2}=1 . c_{1}$ and $c_{2}$ are the intensity means of $\mu_{0}$ inside $C$ and outside $C$. In order to solve energy minimization problem, level set [17-19] methods is used, in which level set function $\phi(\mathrm{x}, \mathrm{y})$ is used in place of unknown curve. The problem of minimization can be solved by taking Euler-Lagrange equation and also by updating level set function $\phi(\mathrm{x}, \mathrm{y})$ by gradient descent method

$$
\frac{\partial \phi}{\partial t}=\delta_{\varepsilon}(\phi)\left[\mu \operatorname{div}\left(\frac{\nabla \phi}{|\nabla \phi|}\right)-\lambda_{1}\left(u_{0}-c_{1}\right)^{2}+\lambda_{2}\left(u_{0}-c_{2}\right)^{2}\right]
$$

Here, $c_{1}$ and $c_{2}$ updates at each iteration by 


$$
\begin{gathered}
c_{1}(\phi)=\frac{\int_{\Omega} u_{0}(x, y) H_{\varepsilon}(\phi(x, y)) d x d y}{\int_{\Omega} H_{\varepsilon}(\phi(x, y)) d x d y}, \\
c_{2}(\phi)=\frac{\int_{\Omega} u_{0}(x, y)\left(1-H_{\varepsilon}(\phi(x, y))\right) d x d y}{\int_{\Omega}\left(1-H_{\varepsilon}(\phi(x, y))\right) d x d y}
\end{gathered}
$$

We have observed that chanvese model and Bhattacharya model are not useful for texture images, where as Gabor based chanvese model works well for texture images but it is not useful for images which have intensity inhomogeneity. Novel structure method only uses LST for edge enhancement.So that these algorithm are not giving better accuracy of texture as well as images which have intensity inhomogeneity. We need to develop some novel technique which give better accuracy of various type of images. Therefore, the objective of the paper to segment the images having following challenges.

- To segment objects from images with constraints of availability of texture region and intensity inhomogeneity simultaneously.

- To improve overall accuracy of active contour based segmentation algorithm by incorporating DOG and LST information inactive contour formulation.

In proposed work, we have developd hybrid model to improve accuracy of various type of images which is explained in next section.

\section{PROPOSED ALGORITHM}

In the proposed method we are using Difference of Gaussians (DOG). The DOG can be explained as below. DOG is basically featuring enhancement in which we can get less obscured (blurred) image from the real image. Blurring using Gaussian kernel repress only high frequency spatial data. DOG is a BPF (band pass filter) that repress all spatial frequency except handful of spatial frequencies that are falls in real gray scale image. The principle advantage DOG offers is that it can provide better edge visibility. One more advantage that DOG provides is the removal of noise compare to other algorithms. Also, it is very fast as far as computational complexity is concerned.

The overall algorithm as follows: Determine DOG Kernal: To determine the kernel, the variance plays the important role. If ratio between two of kernel is large, image blurring would be more. Therefore in proposed model, the size of image is used to calculate the kernel variance.

$$
\begin{aligned}
& G \sigma_{1}=I(x, y) \otimes G \sigma_{1}(x, y) \\
& G \sigma_{k}=I(x, y) \otimes G \sigma_{k}(x, y)
\end{aligned}
$$

Calculate Edges using DOG: DOG Kernels are used to calculate the edges of the image by convolving image with DOG kernels as shown in (8):

$$
\sigma_{1}=k \sigma
$$

Calculate local structure tensor: Medical images can be considered as region of similar texture [20]. Therefore to present its orientation, Structure tensor plays an important role. The structure tensor can be obtained by calculating image edges. In the proposed model, the edge obtained using DOG are used in the calculation of Linear Structure Tensor.

$$
\begin{aligned}
& D_{\sigma, k}(x)=G_{\sigma}(x)-G_{k \sigma}(x) \\
& D_{\sigma, k}(x)=(k-1) \sigma^{2} \nabla^{2} G_{\sigma, k \sigma}
\end{aligned}
$$

Apply Active Contour model [21-23] over LST image: Later the LST based orientation Information is used as an external force in ACM model. The equation (10) can be rewritten using LST as shown in (11). 


$$
\frac{\partial \phi}{\partial t}=\delta_{\varepsilon}(\phi)\left[\mu \operatorname{div}\left(\frac{\nabla \phi}{|\nabla \phi|}\right)-\lambda_{1}\left(D_{\sigma, k}(x)-c_{1}\right)^{2}+\lambda_{2}\left(D_{\sigma, k}(x)-c_{2}\right)^{2}\right]
$$

\section{EXPERIMENT RESULTS AND ANALYSIS}

For accuracy representation of proposed model, we can use Dice Similarity Coefficient (DSC), which can be defined as follow:

\section{$\mathrm{DSC}=2 \mathrm{TP} /(\mathrm{FP}+2 \mathrm{TP}+\mathrm{FN})$}

Where, True Positive=Number of pixels that detected correctly.

False positive=Number of pixels detected as a member of segmented image but not in ground truth image.

False negative-Number of pixels that are not detected but part of ground truth image. We have immplemented this proposed algorithm for texture images, noisy images blur images and images which have intensity inhomogeneity. These images were taken from berkely database [24]. Figures 1 to 6 shows that proposed algorithm gives better edge visibility compared to novel structure tensor based Chanvese model [8]. We have compared the proposed model with chanvese model, Bhattacharya model, gabor based chanvese model and Linear structure tensor based chanvese model for various types of images. It is proven that we got the best accuracy compared to other existing models which is shown in Table 1.

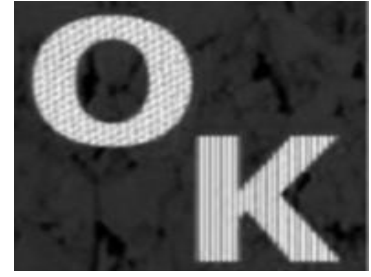

(a)

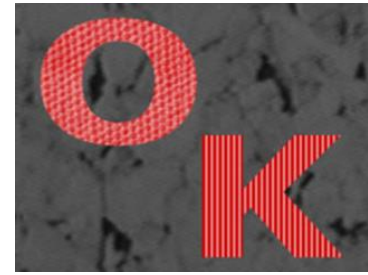

(b)

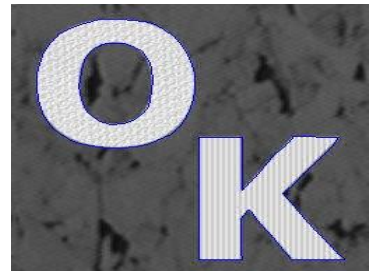

(c)

Figure 1. (a) Original, (b) NSTCV segmented image, (c) proposed model

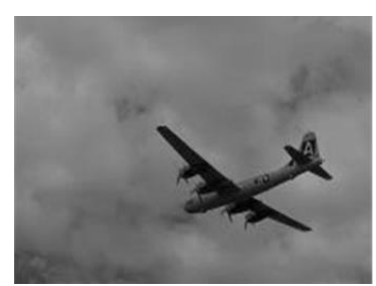

(a)

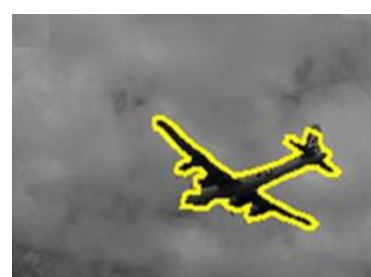

(b)

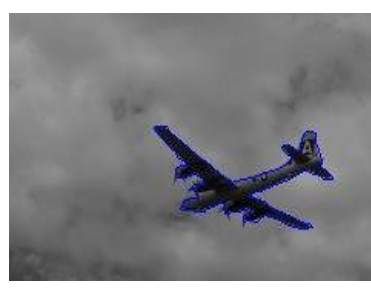

(c)

Figure 2. (a) Original, (b) NSTCV segmented image, (c) proposed model

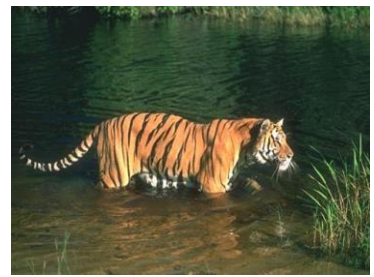

(a)

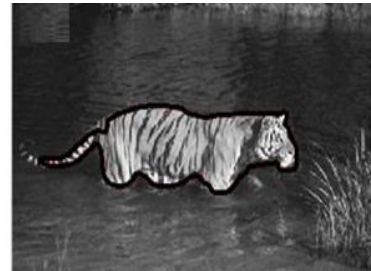

(b)

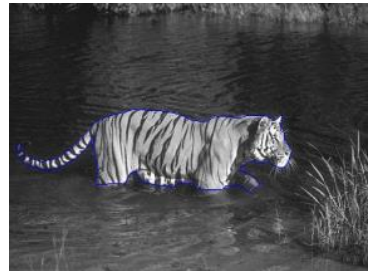

(c)

Figure 3. (a) Original, (b) NSTCV segmented image, (c) proposed model 


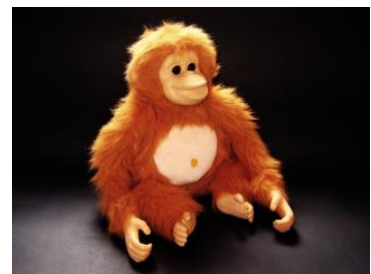

(a)

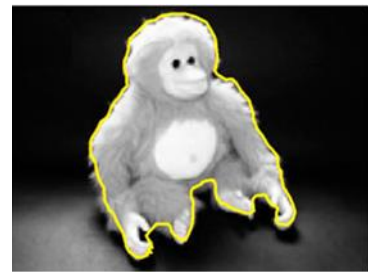

(b)

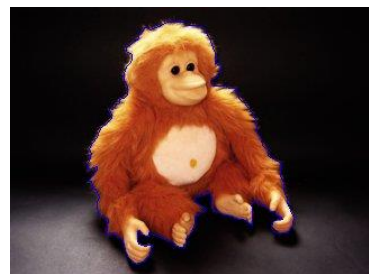

(c)

Figure 4. (a) Original, (b) NSTCV segmented image, (c) proposed model

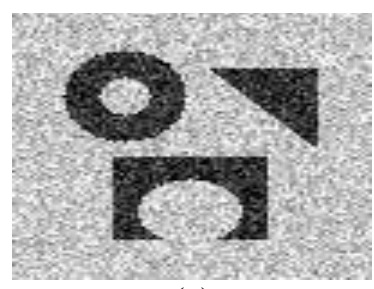

(a)

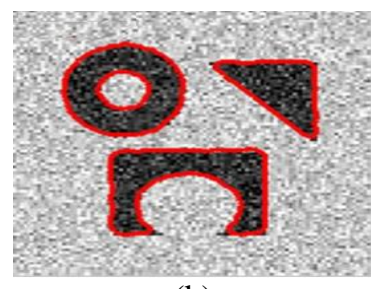

(b)

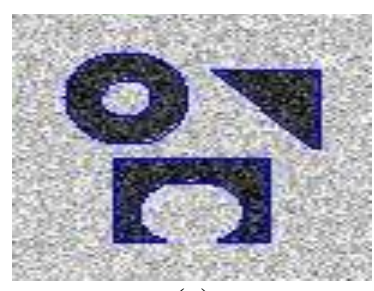

(c)

Figure 5. (a) Original, (b) NSTCV segmented image, (c) proposed model

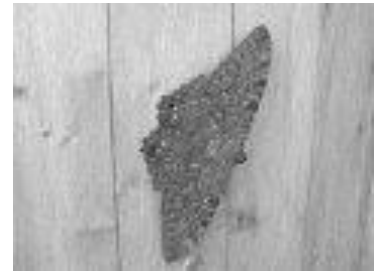

(a)

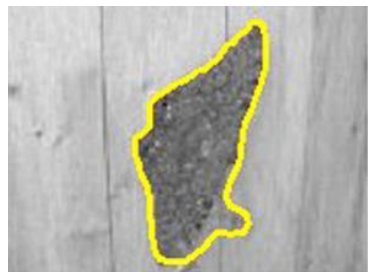

(b)

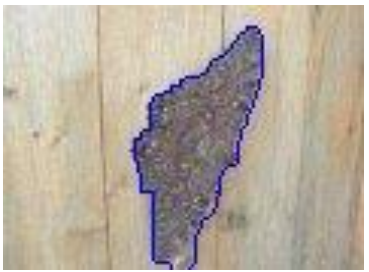

(c)

Figure 6. (a) Original, (b) NSTCV segmented image, (c) proposed model

Table 1 represents the accuracy of proposed Algorithm. We achieve average 99\% accuracy of segmentation. CV Model [25] fails to segment texture as well as images having intensity inhomogeneity. Bhattacharya model [26] also not able to segment some images due to texture regions as well as noise within the image. Gabor and LST based ACM are capable to segment images. However, proposed algorithm outperforms these models and obtained 99\% accuracy amongst all images by using DOG based edge information in LST.

Table 1. Comparison of accuracy for various model [8, 25-27].

\begin{tabular}{cccccc}
\hline $\begin{array}{c}\text { Image } \\
\text { Original } \\
\text { figure }\end{array}$ & $\begin{array}{c}\text { Chan-Vese } \\
\text { Model }\end{array}$ & $\begin{array}{c}\text { Bhattacharya } \\
\text { gradient flow } \\
\text { Model }\end{array}$ & $\begin{array}{c}\text { Gabor based } \\
\text { chanvese model }\end{array}$ & $\begin{array}{c}\text { Novel Structure tensor } \\
\text { chanvese model } \\
\text { Figure 1 (b) to Figure 6(b) }\end{array}$ & $\begin{array}{c}\text { Proposed Model } \\
\text { Figure 1(c) to Figure 6(c) }\end{array}$ \\
\hline 1 & 99.43 & Failed & 99.20 & 98.43 & 99.98 \\
2 & 96.18 & 94.07 & 95.71 & 96.18 & 99.73 \\
3 & Failed & 96.29 & 95.46 & 98.01 & 99.99 \\
4 & Failed & 90.18 & 99.10 & 99.20 & 99.97 \\
5 & 99.48 & Failed & 99.51 & 99.46 & 99.87 \\
6 & 96.64 & 94.69 & 93.85 & 96.64 & 99.26 \\
\hline
\end{tabular}

\section{CONCLUSION}

There are various methods shown for comparision. Chanvese and bhattacharya model are not so good when texture type of images are given as input. Gabor based chanvese model can solve the problem of texture images but when intensity inhomogeneity is there ,it cannot give proper result. Novel structure tensorchanvese model works well forimages with intensityinhomogeneity. A LST is a good descriptor and 
also mathematically easy to implement. But specifically for texture image Gaussian filter will create some problems. In this paper LST has been modified for removing edge dislocationTexture smotthing can also been done with this method. But the biggest advantage of proposed method is it uses DOG alongwith LST.

We can also see the accuracy of the proposed model is better than almost any other model in almost all the cases. The advantage of DOG is that it is less prone to noise. This paper proposed structure tensor using DOG kernel .The orientation information obtained from Linear Structure Tensor is used in active contour model to obtain segmentation. We have shown that the proposed model works effectively for various types of images. We also obtained better Edge enhancement using this method.

\section{REFERENCES}

[1] C. Li, C. Kao, J. Gore, and Z. Ding. "Implicit active contour driven by local binary fitting energy," IEEE Conference on Computer Vision and Pattern Recognition, pp. 1-7, 2007.

[2] V. Caselles, F. Catte, T. Coll, F. Dibos, "A geometric model for active contours in image processing," Numer. Math., Vol. 66, No.1, pp. 1-31, 1993.

[3] M. Kass, A. Witkin, D. Terzopoulos, "Snakes: active contour models", Int. J. Comput. Vision, Vol. 1,No. 4,pp. 321-331, 1987.

[4] T. F. Chan, L. A. Vese, "Active contours without edges", IEEE Trans. Image Process., Vol.10, No.2, pp. 266-277, 2001.

[5] D. E. Maroulis, M. A. Savelonas, D. K. Iakovidis, S. A. Karkanis, N. Dimitropoulos, "Variable background active contour model for computer-aided delineation of nodules in thyroid ultrasound images", IEEE Trans. Inf. Technol. Biomed., Vol.11, No. 5, pp. 537-543, 2007.

[6] B. Sandberg, T. Chan, and L. Vese, "A level-set and Gabor-based active contour algorithm for segmenting textured images", Technical Report 39, Mathematical Department, UCLA, Los Angeles, 2002.

[7] Xiao-Feng Wang De-Shuang Huang HuanXu, "An efficient local Chan-Vese model for image segmentation", Pattern Recognition, No.43, pp.603-618, 2010.

[8] HirenMewada,Rahul Patel \& SuparvaPatniak, "A Novel Structure tensor Modulated Chan-Vese Model for texture image segmentation", The computer Journal, Vol.12, pp.1-17, 2014.

[9] S. Osher, J. A. Sethian, "Fronts propagating with curvature-dependent speed: algorithms based on Hamilton-Jacobi formulations", J. Comput. Phys., Vol. 79, No. 1, pp. 12-49, 1988.

[10] D. Mumford, J. Shah, "Optimal approximation by piecewise smooth functions and associated variational problems", Commun. Pure Appl. Math., Vol. 42, pp. 577-685, 1989.

[11] T. Brox, J. Weickert, B. Burgeth, and P. MrAazek. "Nonlinear structure tensors", Image and Vision Computing, Vol.24, No.1, pp.41-55, 2006.

[12] Do, M. and Vetterli, M.,"Wavelet-based texture retrievalusing generalized Gaussian density and Kullback-Leibler distance", IEEE Trans. Image Process., Vol.11, pp.146-158, 2002.

[13] Hofmann, T., Puzicha, J. and Buhmann, J. M." "Unsupervised texture segmentation in a deterministic annealing framework”.IEEE Trans. Pattern Anal. Mach. Intell., Vol.20, pp.803-818, 1998.

[14] A. Gelas, O. Bernard, D. Friboulet, R. Prost, "Compactly supported radial basis functions based collocation method for level-set evolution in image segmentation”, IEEE Trans. Image Process., Vol. 16, No.7, pp. 1873-1887, 2007.

[15] Alpert, S., Galun, M., Brandt, A. and Basri, R. “ Image segmentation by probabilistic bottom-up aggregation and cue integration”, IEEE Trans. Pattern Anal. Mach. Intell., Vol.34, pp.315-327, 2012.

[16] Dubuisson, S. "The Computation of Bhattacharyya Distance Between the Histograms without Histograms", 2ndInt. Conf. on Image Processing Theory Tools and Applications, Paris, France, pp. 373-378. IEEE France Section, July7-10, 2010.

[17] Feddern, C., Weickert, J. and Burgeth, B., "Level-Set Methods for Tensor-Valued Images. Proc", 2nd IEEE Workshop on Variational, Geometric and Level Set Methods in ComputerVision, France, pp. 65-72, October10-12, 2003.

[18] Xiao, J., Xu, L., Yi, B. and Xie, W., "The Improvementof C-V Level Set Method for Image Segmentation”, Int. Conf. on Computer Science and Software Engineering, China, pp. 1106-1109. IEEE Computer Society, December $12-14,2008$

[19] Bigun, J. and Grandlund, G., "Optimal Orientation Detection of Linear Symmetry", 2nd IEEE Workshop onVariational, Geometric and Level Set Methods in ComputerVision, pp. 65-72. IEEE Computer Society, London, UK, June 8-11, 1987.

[20] Conners, R. and Harlow, C. "“A theoretical comparison oftexture algorithms”. IEEE Trans. Pattern Anal. Mach. Intell., Vol. 2, pp. 204-222, 1980.

[21] Tatu, A. and Bansal, S.," A novel active contour model fortexture image segmentation”, CORR, abs/1306.6726, 2013.

[22] Michailovich, O., Rathi, Y. and Tannenbaum, A. ,"Imagesegmentation using active contours driven by the Bhattacharyya gradient flow",. IEEE Trans. Image Process., Vol.16, pp.2787-2801, 2007.

[23] Lee, S., Abott, A., Clark, N. and Araman, P., "Active Contours on Statistical Manifolds and Texture Segmentation.Proc”, IEEE Int. Conf. on Image Processing, Italy, IEEE Signal Processing, pp. 828-831. September 11-14, 2005.

[24] Berkely image database https://www2.eecs.berkeley.edu/Research/Projects/CS/vision/bsds/ 
[25] A. Tsai, A. Yezzi, A. S. Willsky, "Curve evolution implementation of the Mumford-Shah functional for image segmentation, de-noising, interpolation, and magnification", IEEE Trans. Image Process., Vol. 10, No.8, pp. 1169-1186, 2001.

[26] Chen, M. and Strobl, J., "Multispectral textured image segmentation using a multi-resolution fuzzy Markov random field model on variable scales in the wavelet domain", Int. J. Remote Sens., Vol. 34, pp.4550-4569, 2013.

[27] T. Brox. "From pixels to regions: Partial differential equations in image analysis", PhD Thesis, Mathematical Image Analysis Group, Department of Mathematics and Computer Science Saarland University, Germany, 2005.

\section{BIOGRAPHIES OF AUTHORS}

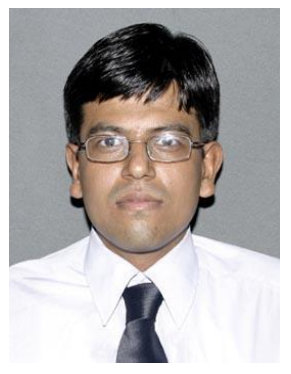

Brijesh N Shah is a PhD Scholar with the Department of Electronics and Communication Engineering at C S Patel Institute of Technology-Charotar University of Science and Technology, Changa, Anand, Gujarat, India. He received Bachelor of Engineering degree in Electronics and Communication Engineering from Saurashtra University and Master of Engineering degree in Electronics and Communication Engineering from Dharmsinh Desai University. He is currently working towards his Ph.D degree at Department of Electronics and Communication Engineering, Charotar University of Science and Technology. His current research interest lies in Image Processing

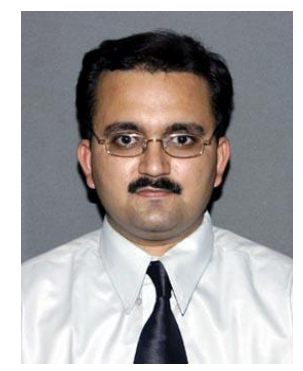

Dr. Jaymin K Bhalani is Professor with the Department of Electronics and Communication Engineering at Babaria Institute of Technology-Varnama, Vadodara, Gujarat, India. His current research interests are in Image Processing, Wireless Communication Systemsand Signal Processing. He has published several papers in national/international conferences and international journals. He received Master of Engineering degree in Electronics and Communication Engineering with Specialization of Communication Systems Engineering from Gujarat University, India. He received Ph.D. degree from MSU, Vadodara. 